\title{
Mejoramiento de la comprensión auditiva en portugués como LE en contextos comunicativos mediados por la tecnología*
}

\author{
Improvement of listening comprehension in Portuguese as FL \\ in technology-mediated communication contexts \\ Melhora da compreensão oral em português como LE \\ em contextos comunicativos mediatizados pela tecnologia
}

\author{
Natalia Asenjo Zapata, ${ }^{a}$ Anita Ferreira Cabrera. ${ }^{b}$ \\ a Universidad de Concepción, Chile. Fono: 78776192. Correo electrónico: nataliaasenjo@udec.cl \\ b Universidad de Concepción, Chile. Fono: 2203776. Correo electrónico: aferreir@udec.cl
}

\begin{abstract}
RESUMEN
La comprensión auditiva sigue siendo una destreza poco estudiada. En este contexto, las nuevas tecnologías se presentan como herramientas que favorecen su práctica y desarrollo. Algunas lenguas, que antes no siempre eran accesibles, actualmente son enseñadas y aprendidas apoyadas por la tecnología, como el portugués. El propósito de este trabajo es determinar el mejoramiento de la comprensión auditiva del portugués como lengua extranjera en aprendientes hispanohablantes, mediante la realización de tareas cooperativas a través del uso de videoblog, foro y videocomunicación. Específicamente, se analizan los aspectos de procesamiento y comprensión, tanto de la información explícita como de la implícita. Para este fin, se diseñó un estudio experimental con grupo control y medición antes y después del período de intervención. Los resultados obtenidos muestran que el uso de la comunicación mediada por la tecnología favorece el desarrollo de la comprensión auditiva, comparado con la forma más tradicional de práctica de esta destreza.
\end{abstract}

Palabras clave: comprensión auditiva, enseñanza del portugués como lengua extranjera, CMC, CALL.

\begin{abstract}
Listening comprehension has remained as a less-studied skill. In this context, new technologies are considered effective tools in its practice and development. Thus, it has been observed that languages which seemed to have a harder access, like Portuguese, are currently taught and learnt through these technologies. The aim of this work is to determine the improvement of listening comprehension in Portuguese as a foreign language on Spanish-speaking learners by carrying out cooperative tasks through the use of video blogs, forums and video communication. Specifically, the processing and understanding of explicit and implicit information. In order to comply this, an experimental study has been designed with a control group to measure before and after the intervention process. The obtained results confirm that the use of technology stimulates and benefits the development of listening comprehension, in comparison with the most traditional form of practice of this skill.
\end{abstract}

Key words: listening comprehension, teaching of Portuguese as a foreign language, CMC, CALL.

\section{RESUMO}

A compreensão oral continua uma habilidade pouco estudada. Neste contexto, as novas tecnologias apresentamse como ferramentas que favorecem sua prática e desenvolvimento. Algumas línguas, como o português, antes

La investigación que se presenta en este artículo fue realizada en el contexto del proyecto de investigación FONDECYT 1110812: "Un Sistema Tutorial Inteligente para la Focalización en la Forma en la Enseñanza de Lenguas Extranjeras", que dirige la Dra. Anita Ferreira Cabrera. 
não tão acessíveis, atualmente são ensinadas e aprendidas apoiadas pela tecnologia. O objetivo deste estudo é determinar a melhora da compreensão oral do português como língua estrangeira de alunos falantes do espanhol, mediante a realização de tarefas cooperativas por meio de videoblog, fórum e vídeo comunicação. Especificamente, analisam-se os aspectos de processamento e compreensão tanto da informação explicita como da implícita. Para tal, foi elaborado um estudo experimental com grupo controle e medições pré- e pós-teste. Resultados obtidos mostram que o uso da comunicação mediatizada pela tecnologia favorece o desenvolvimento da compreensão oral, em comparação com forma mais tradicional de prática de esta habilidade.

Palavras chave: compreensão oral, ensino do português como língua estrangeira, CMC, CALL.

\section{INTRODUCCIÓN}

Actualmente, el portugués es la cuarta lengua más aprendida como LE en el mundo (Albuquerque y Esperança, 2010), siendo los hispanohablantes los más interesados en aprenderla (Almeida Filho, 2004). Uno de los aspectos más complejos de desarrollar en esta lengua es la comprensión auditiva, especialmente, para aprendientes hispanohablantes (Holton, 1954). Esta dificultad se acrecienta debido a los pocos estudios realizados de manera consistente en portugués como lengua extranjera (Pinto, 2010).

Hasta hace algún tiempo, la comprensión auditiva consistía en escuchar repetidamente una cinta de audio. Era un proceso iterativo, con material no auténtico ni significativo para los aprendientes (Jones, 2008). Las actividades se limitaban a la comprobación de la comprensión sólo mediante respuestas correctas (Meskill, 1996). Afortunadamente, este paradigma ha ido cambiando. Una muestra ha sido pasar de la consideración de esta destreza como un proceso pasivo a uno activo (Vandergrift, 2004; Lynch, 2002; Peris, 1991). Esto es así debido a los conocimientos que debe manejar el oyente para comprender un mensaje oral (Anderson \& Lynch, 1988). Recientemente, la habilidad de la comprensión auditiva se analiza como un proceso multifisiológico y socio cognitivo complejo, en el cual la interacción entre los participantes es fundamental (Meskill, 1996).

En materia tecnológica, los distintos recursos que entrega la Comunicación Mediada por Computador (CMC) permiten trabajar con textos orales auténticos y contextualizados. $\mathrm{Su}$ uso favorece, especialmente, el desarrollo y la práctica de la comprensión auditiva. Según señala Meskill (1996), esto ha sido demostrado en diversos estudios, como los conducidos por Mueller (1980) y Omaggio (1980) (Cit. en Meskil, 1996).

El presente artículo aborda la problemática de cómo mejorar el desarrollo de la comprensión auditiva del portugués (en su variante brasileña) como lengua extranjera en hispanohablantes, a través del uso del videoblog, el foro y la videocomunicación. Se entenderá por comprensión auditiva lo planteado por Buck (2001) en el Constructo Estandarizado para la Comprensión Auditiva (del inglés, Default Listening Construct). Este constructo considera no sólo las competencias lingüísticas básicas, sino también otras características de la lengua, como los conocimientos pragmáticos y discursivos para la realización de inferencias.

\section{MARCO TEÓRICO}

A continuación se presentan los fundamentos teóricos que sustentan la problemática en estudio. Éstos se sitúan en el ámbito de la Adquisición de Lenguas Extranjeras y la Interdisciplina CALL, a partir de la integración de recursos tecnológicos en metodologías de enseñanza de lenguas basadas en tareas cooperativas. 


\subsection{COMPRENSIÓN AUDITIVA (CA)}

Los avances tecnológicos de las últimas décadas han producido un cambio en la enseñanza de esta destreza. Se ha pasado de escuchar una cinta varias veces al uso de tecnologías audiovisuales que enriquecen el proceso de aprendizaje, haciéndolo más motivador (Jones, 2008). Es así como hoy en día se busca demostrar los beneficios del uso de CMC para el desarrollo de la CA, ya que entrega un input auténtico, contextualizado y en tiempo real.

La CA continúa siendo una destreza poco estudiada, dado que no se la puede medir directamente y se la aprecia a partir del desempeño del oyente al realizar una determinada tarea. Es, por lo tanto, difícil evaluarla y aprenderla (Vandergrift, 2004). Sólo desde hace poco tiempo ha comenzado a ser considerada una destreza activa (Vandergrift, 2004; Lynch, 2002; Peris, 1991), debido a la cantidad de conocimientos que el oyente debe accionar para poder comprender un mensaje (Anderson \& Lynch, 1988). El reconocimiento de fonemas, tonos, repeticiones, palabras nuevas, gestos, distancia entre los interlocutores y contexto son sólo algunos de los aspectos implicados en su enseñanza.

De este modo, esta destreza involucra un complejo procedimiento de interpretación e interacción (Buck, 2001), el cual consta de dos procesos complementarios: el procesamiento sintético (del inglés, top-down) y el analítico (del inglés, bottom-up). Cuando un oyente está haciendo uso de su conocimiento previo y contextual, es decir, desde la realidad del mundo al mensaje, favorece el procesamiento analítico. En cambio, cuando el oyente parte desde el mensaje, reconociendo fonemas, luego palabras, después oraciones y posteriormente textos, está utilizando el procesamiento sintético (Sato, 2011; Andrighetti, 2009; Johnson, 2008; Vandergrift, 2007, 2004; Brown, 2006; Córdova, Coto y Ramírez, 2005; Buck, 2001).

Vandergrift (2011) propone, además, considerar las tres fases de procesamiento propuestas por Anderson (Cit. en Vandergrift, 2011: 457): percepción, análisis y utilización. A pesar de que se pueden considerar también para las otras destrezas, este autor las adapta para trabajar de manera conjunta con los dos procesos anteriores (analítico y sintético). Se obtiene, así, un modelo interconectado para comprender, obtener un significado y dar una respuesta adecuada a la situación comunicativa correspondiente. La figura que se presenta a continuación muestra cómo se procesa la información durante la comprensión de un mensaje oral.

Figura $N^{\circ} 1$. Procesamiento comprensión auditiva

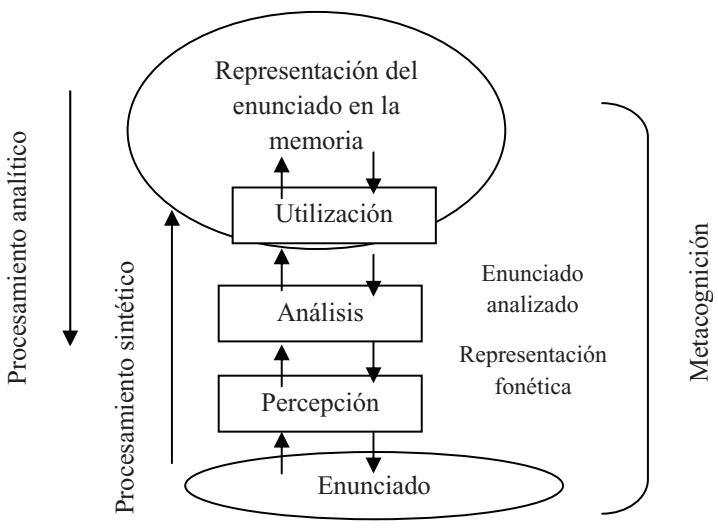

Fuente: traducido de Vandergrift (2011). 
En un primer momento, el oyente reconoce las categorías de sonidos, pausas y énfasis, y los mantiene por un corto período en la memoria de trabajo (percepción - procesamiento sintético). Luego, recupera de su memoria a largo plazo las posibles palabras que se corresponderían con los sonidos escuchados, les da un posible significado y los retiene en la memoria de trabajo mientras se procesa el nuevo input (análisis). Finalmente, el oyente usa y relaciona la información extralingüística para interpretar los significados que ha retenido en su memoria (utilización - procesamiento analítico).

Todo el proceso es muy rápido y en la Lengua Materna (LM) ocurre de manera mecánica, sin que los oyentes sean conscientes de esto. Sin embargo, en la LE, los estudiantes deben aprender a usarlos, ya que son necesarios para las interacciones del día a día para las cuales se los prepara. Saber una lengua y dominar su sistema fonético/fonológico, sintáctico o semántico no es suficiente si están descontextualizados. Si falla uno u otro, el mensaje será incomprensible. Por lo tanto, la enseñanza de la CA de una lengua extranjera debe abarcar los niveles fonético, fonológico, prosódico, léxico, semántico, sintáctico y pragmático, con sus respectivas microdestrezas.

\subsubsection{Constructo Estandarizado para la Comprensión Auditiva}

Antes de comenzar a diseñar pruebas, tareas o actividades destinadas a la evaluación o práctica de la CA, Buck (2001) propone definir el constructo con el cual se debe trabajar. Éste corresponde al aspecto que se va a medir o evaluar. Así se deben determinar las habilidades, las microdestrezas y el proceso de evaluación. Esta definición permite coherencia teórica y práctica a la hora de desarrollar esta destreza.

El Constructo Estandarizado para la Comprensión Auditiva (del inglés, Default Listening Construct), según su propio autor (Buck, 2001), es el mejor acuerdo entre el rigor teórico, la utilidad práctica y el retorno del trabajo realizado. En él, la comprensión auditiva es estudiada y trabajada desde un amplio punto de vista lingüístico. Es decir, se incluyen no sólo aspectos fonológicos, léxicos o sintácticos, sino también los otros mecanismos que los emisores usan para expresar significados, como por ejemplo: entonación, tono de voz, énfasis, elección de palabras, estructura discursiva, etc. Dicha propuesta considera ir más allá de los significados literales, pero sin incluir toda la gama de significados implícitos.

De esta forma, la CA se configura como la habilidad para:

- Procesar muestras de lenguaje oral auténtico, automáticamente y en tiempo real.

- Entender la información lingüística que aparece de forma inequívoca en el texto.

- Hacer inferencias que aparezcan inequívocamente implicadas en el contenido del texto.

Debido a que los sujetos de la presente investigación son hispanohablantes que aprenden portugués como LE, se estima adecuado partir desde el inicio con la presentación de material auténtico y muestras reales de lengua (velocidad de habla normal, modismos, elisiones, etc.), y la enseñanza de inferencias, que aparezcan claramente y sin lugar a dudas en el enunciado. Esta decisión se sustenta en la velocidad y el tipo de aprendizaje, el cual es rápido y, por lo tanto, requiere materiales ágiles, desafiantes y de enseñanza temática e interdisciplinar (Bison, 1994; Widdowson, 1991). 


\subsection{COMUNICACIÓN MEDIADA POR COMPUTADOR (CMC)}

La Comunicación Mediada por Computador (del inglés, Computer Mediated Communication, CMC) es definida como "el tipo de comunicación humana lograda a través de o con la ayuda de computadores" (Quintanilla y Ferreira, 2010: 218), ya sea de manera sincrónica o asíncrona, dependiendo de si la interacción comunicativa se lleva o no a cabo en tiempo real. Existen diversos recursos que posibilitan la CMC, algunos de comunicación sincrónica, y otros, asíncrona. A continuación se describen los más relevantes para el presente estudio.

\subsubsection{Recursos sincrónicos}

Los recursos sincrónicos, como el chat, ayudan a los aprendientes a considerarse parte de una comunidad de aprendizaje, haciéndolos sentir más motivados y menos aislados (White, 2003). Con este tipo de herramientas se promueve el uso de una interacción más espontánea e interactiva, similar a lo que ocurre en una comunicación cara a cara.

\subsubsection{Videocomunicación}

La videocomunicación puede situarse dentro de los denominados chat sincrónicos. Según Lamy \& Hampel (2007), fue el primer recurso utilizado para la enseñanza de lengua, ya que ofrece una plataforma ideal para simular la comunicación oral.

Con el uso de esta herramienta la comunicación se lleva a cabo en dos direcciones, mediante el uso de imagen y voz. Vine y Ferreira (2012) mencionan que la importancia de este recurso radica en la posibilidad de simular lo que ocurriría en una interacción oral cara a cara. Esto ayudaría a los estudiantes en su proceso de aprendizaje, y consecuentemente, desarrollaría su competencia comunicativa en la lengua meta.

Asimismo, Jauregi, Canto y Gómez (2010) afirman que las interacciones a través de la videocomunicación son altamente contextualizadas y entregan pistas paralingüísticas (como los gestos, el contacto visual o el tono de voz). Además presentan el dinamismo, la flexibilidad y la espontaneidad de la oralidad.

Para los propósitos del presente estudio, se hace uso de Skype, el cual presenta ventajas en la calidad de imagen y sonido. Además, es gratuito, fácil y rápido de descargar, posee una interface amigable para el usuario y posibilita guardar un registro de las conversaciones.

\subsubsection{Recursos asíncronos}

Los recursos asíncronos promueven la reflexión por parte de los estudiantes, ya que permiten pensar las respuestas o comentarios. Son flexibles en tiempo y lugar. Se puede ingresar a ellos en cualquier sitio, sin un horario determinado y entregan la posibilidad de una posterior revisión (White, 2003).

\subsubsection{Videoblog}

En el presente estudio, el blog se configura como un videoblog. En vez de contener sólo textos o imágenes, aparecen además videos para ser comentados como tópico central. Una 
de las ventajas de esta aplicación es su flexibilidad, permitiendo adecuarse a las necesidades del contexto de enseñanza/aprendizaje en el cual se va a aplicar.

\subsubsection{Foro}

El foro comparte algunas de las características del blog, pero su finalidad es promover la discusión, el debate, el intercambio de opiniones, la reflexión y el consenso de ideas (Ornelas, 2007; Adrián y Gros, 2004; Arango, 2003). Así, se establece como un lugar de reunión que permite que sus participantes construyan el conocimiento a partir de los distintos diálogos que se desarrollan en él. Desde la perspectiva de la enseñanza, fomenta la colaboración entre los estudiantes como apoyo a su proceso de aprendizaje. En el presente estudio, este recurso se configura como un espacio en el que los aprendientes practiquen sus habilidades de escritura y lectura, discutiendo, resolviendo y comparando soluciones, tanto entre los propios aprendientes como con el profesor (Ornelas, 2007).

\subsubsection{Blended Learning o Modalidad Semipresencial}

Todos los recursos de CMC (sincrónicos y asincrónicos) se utilizan en una modalidad combinada o blended learning. Dicha modalidad reúne aspectos o características de la presencialidad y la no presencialidad que se han observado favorables para la enseñanza. Entre los beneficios con los que cuenta se puede encontrar una buena distribución de los recursos de acuerdo con las características de cada habilidad lingüística. Esto, articulado según los objetivos de enseñanza, permite un mejor desarrollo de dichas habilidades en los aprendientes (Morales y Ferreira, 2008). Asimismo, promueve el aprendizaje de los estudiantes según su propio ritmo, característica relevante del aprendizaje a distancia, junto con la contextualización típica de la enseñanza presencial.

$\mathrm{Su}$ efectividad en el aprendizaje de lenguas ha sido validada por diversos estudios, como los realizados por García et al. (2012), Vine y Ferreira (2012), Quintanilla y Ferreira (2010), Morales y Ferreira (2008) y Ferreira (2007). En ellos, su utilización para la enseñanza del inglés y español como L2 y LE apoyó el desarrollo de las habilidades lingüísticas y de la interacción de los aprendientes.

Debido a sus resultados positivos y a las diversas ventajas antes mencionadas, se ha decidido trabajar con esta modalidad. Mientras en las clases presenciales se focalizaron aspectos problemáticos, resolución de dudas y evaluación del proceso de aprendizaje, en las clases mediadas por computador (no presenciales) se fomentó el desarrollo de la comprensión auditiva.

\subsubsection{Enfoques de enseñanza de lenguas}

Los enfoques de enseñanza sobre los cuales se basa la presente investigación forman parte de los denominados enfoques comunicativos. A continuación se describen los dos enfoques más relevantes para este estudio.

\subsubsection{Enfoque por Tareas}

El Enfoque por Tareas no considera la lengua como objeto de estudio en sí mismo, sino como una herramienta cuya función es permitir la interacción entre personas mediante 
la práctica y el desarrollo de la competencia comunicativa de los aprendientes (Estaire, 2009). Es decir, se aprende la lengua a medida que se realizan ciertas tareas, las cuales se relacionan con actividades que se pueden encontrar fuera de la sala de clases. El aprendiente es un agente activo y el creador de su propio proceso de aprendizaje, y el profesor pasa a desempeñar el papel de mediador y facilitador de la lengua (Quintanilla y Ferreira, 2010).

Cada tarea consta de tres fases para su realización. La pre tarea consiste en actividades de "precalentamiento"; la tarea propiamente tal, que abarca actividades de comprensión, expresión e interacción, y en las que el profesor permite mayor protagonismo de los aprendientes; y la post tarea, momento de monitoreo, reflexión sobre el aprendizaje y atención en la forma de las posibles dificultades que pudieran haber aparecido (Livingstone y Ferreira, 2010; Quintanilla y Ferreira, 2010).

Con respecto al uso de tareas en ambientes tecnológicos, los computadores se presentan como herramientas efectivas para su implementación (Doughty \& Long, 2003). Permiten un mayor y mejor acceso a material auténtico, a interacciones con nativos y a contextos de uso de lengua variados. Además, la integración de algunos de los recursos que posibilitan la comunicación mediada por computador favorecen la construcción del propio conocimiento en la lengua meta, a medida que se interactúa y coopera con otros (nativos y no nativos). Esto sucede sin perder de vista el aprendizaje individualizado, en el cual el estudiante avanza a su propio ritmo, con confianza y en un ambiente controlado.

Para los propósitos de este estudio, se usa el tipo de tareas de intercambio de opinión (del inglés, Opinion Exchange Task), de acuerdo a la clasificación realizada por Pica, Kanagy \& Falodun (1993). En este tipo de tareas, los aprendientes participan en debates e intercambios de ideas, sin que sea necesario llegar a un consenso.

\subsubsection{Aprendizaje Cooperativo de Lenguas}

El Aprendizaje Cooperativo de Lenguas apoya la construcción de conocimiento mediante la colaboración, más que la competición, para alcanzar un objetivo o resultado. Por una parte, el profesor es un mediador y facilitador del aprendizaje. Por otra parte, el estudiante es un participante activo, responsable de la construcción de su propio conocimiento y del de los demás.

Entre las características fundamentales y necesarias para que el Aprendizaje Cooperativo de Lenguas sea efectivo, en primer lugar, cabe mencionar la interdependencia positiva. Ésta es la encargada de promover la idea de compañerismo y de grupo (Johnson y Johnson, 1999). En segundo lugar, se encuentra la responsabilidad individual. Cada estudiante es responsable de su propio aprendizaje y de ayudar a los otros miembros del grupo a aprender. Las interacciones positivas entre ellos favorecen los aspectos antes mencionados, permitiendo evaluar de forma más objetiva y crítica el funcionamiento del grupo.

Las actividades planteadas bajo este enfoque buscan provocar la comunicación para resolver un problema de lengua en conjunto y de forma interactiva. La técnica específica usada en el presente estudio corresponde a la denominada Aprendiendo Juntos (del inglés, Learning Together). Todos los grupos trabajan en una única tarea y buscan tanto el éxito individual como grupal. Es la técnica con más estudios validando sus ventajas y beneficios (Trujillo Sáez, 2002).

Para los objetivos de esta investigación se trabajó con el grupo cooperativo formal. Aunque su duración no es de largo plazo, interactuar algunas semanas en parejas con el 
mismo compañero es tiempo suficiente para que los aprendientes construyan lazos de confianza y camaradería entre ellos. Esto ayuda al fomento en la interdependencia positiva necesaria para la consecución de los objetivos propuestos.

Además, debido a que el apoyo de la tecnología cumple un rol fundamental en la presente investigación, se puede señalar que el computador se configura como un medio natural para la colaboración (Morales y Ferreira, 2008). Cada uno de los recursos, aplicaciones y posibles usos que conlleva, favorecen las interacciones auténticas en la resolución de un problema.

\section{ESTUDIO EXPERIMENTAL}

Con el fin de determinar si el uso integrado del videoblog, el foro y la videocomunicación puede mejorar la comprensión auditiva de portugués como LE en hispanohablantes, se diseñó un estudio experimental, con pre, post test y con grupo control. Se definió la pregunta de investigación: ¿se puede mejorar la comprensión auditiva de hispanohablantes aprendientes de portugués como LE, siguiendo el constructo estandarizado propuesto por Buck (2001), a través de la realización de tareas cooperativas implementadas en videoblog, foro y videocomunicación?

A partir de esta pregunta, se planteó la siguiente hipótesis: el uso de tareas cooperativas implementadas en videoblog, foro y videocomunicación mejora la comprensión auditiva en hispanohablantes que aprenden portugués como LE, siguiendo el constructo estandarizado para la comprensión auditiva propuesto por Buck (2001).

\subsection{METODOLOGÍA}

\subsubsection{Muestra}

La muestra quedó conformada por 16 estudiantes hispanohablantes que cursaban distintas asignaturas en la Universidad Estadual de Campinas - Brasil, y aprendían portugués como LE en el Centro de Estudos de Línguas (CEL) de la misma casa de estudios. Todos los aprendientes eran de nivel básico, respaldado por el proceso selectivo de este centro ${ }^{1}$.

Del total de sujetos participantes, 6 eran mujeres $(37,5 \%)$ y 10 eran hombres $(62,5 \%)$, cuyas edades fluctuaban entre los 21 y 38 años. El promedio de edad del grupo control fue de 28,2 años, mientras que en el grupo experimental fue de 23 años.

Los aprendientes eran de diferentes nacionalidades: argentinos (12,5\%), chilenos $(12,5 \%)$, colombianos $(18,7 \%)$, cubanos $(6,2 \%)$, españoles $(18,7 \%)$, mexicanos $(6,2 \%)$, paraguayos $(6,2 \%)$, peruanos $(12,5 \%)$ y venezolanos $(6,2 \%)$. Además, $50 \%$ eran de pregrado (cursando un semestre de intercambio) y $50 \%$ de postgrado (en su primer semestre de estudios).

Ambos grupos realizaron las mismas tareas cooperativas para el mejoramiento de comprensión auditiva, pero se diferenciaron en la metodología de trabajo. La muestra se distribuyó de la siguiente manera:

El CEL al inicio de cada semestre realiza una entrevista personal entre el estudiante y el profesor de la lengua a ser aprendida (en este caso portugués), en la cual se determina el nivel de proficiencia del candidato. 
- Grupo experimental: los aprendientes de este grupo trabajaron en una modalidad semipresencial durante el módulo de intervención con los recursos tecnológicos de videoblog, foro y videocomunicación.

- Grupo control: los aprendientes usaron audios e imágenes impresas (una por audio), correspondientes a las sesiones del período de intervención en una modalidad presencial.

\subsection{PROCEDIMIENTO}

Para llevar a cabo el presente estudio se desarrollaron diferentes herramientas de recolección de datos. En un primer momento, y para encontrar posibles problemas que pudieran surgir durante la aplicación, se llevó a cabo el estudio piloto. Este paso fue importante metodológicamente, ya que se hicieron las modificaciones necesarias para el período de intervención.

El segundo instrumento aplicado fue un cuestionario inicial, el cual permitió conocer las características de los aprendientes. Entre ellas destacan: el tiempo de estudio en la lengua, cuáles eran los aspectos que consideraban más difíciles, si hablaban otras lenguas, etc.

En tercer lugar, se desarrolló un pre test, el cual constó de dos partes. La primera consideró un audio de 01:05 minutos de duración de una novela brasileña que se exhibió por televisión abierta en el año 2011. A partir de la audición de dicho material, los estudiantes tenían que responder 8 preguntas abiertas. El uso de este tipo de audios auténticos está en consonancia con lo planteado por Brown (2001), quien señala que de esta forma se trabaja con interacciones orales contextualizadas y que cumplen una función social dentro de la comunidad de la lengua meta. De esta manera, se promueve la comprensión auditiva de la nueva lengua.

En la segunda parte del pre test se les pidió a los aprendientes que escucharan dos oraciones con un matiz más cultural (expresiones populares y de uso cotidiano), y escribieran lo que ellos creían que éstas significaban para poder evaluar la formulación de inferencias a partir de un texto oral, como lo propone Buck (2001) en su constructo. A partir del Constructo Estandarizado para la Comprensión Auditiva, se elaboró la rúbrica que se muestra en la Tabla $\mathrm{N}^{\circ} 1$, cuyos parámetros definidos son:

- Escuchar: prestar atención durante las diferentes actividades de audición.

- Procesar: procesamiento automático de material oral auténtico y en tiempo real.

- Entender: comprensión de la información lingüística que aparece en el texto oral.

- Inferir: realizar inferencias implicadas, inequívocamente, en el contenido del enunciado.

Tabla $N^{\circ} 1$. Rúbrica de evaluación de acuerdo al Constructo Estandarizado para la Comprensión Auditiva

\begin{tabular}{|c|c|c|c|}
\hline \multicolumn{2}{|l|}{ Puntaje Ideal } & Puntaje Obtenido & Porcentaje \\
\hline \multirow{3}{*}{$\begin{array}{l}\text { Parte I: } \\
\text {-Escuchar }(20 \%) \\
\text {-Entender }(20 \%) \\
\text {-Procesar }(20 \%) \\
\text {-Inferir }(20 \%)\end{array}$} & \multirow{3}{*}{80 pts. } & \multirow{3}{*}{$\begin{array}{l}\text { Parte I: } \\
\text {-Escuchar }(20 \%) \\
\text {-Entender }(20 \%) \\
\text {-Procesar }(20 \%) \\
\text {-Inferir }(20 \%)\end{array}$} & 91\%-100\%: Muy competente \\
\hline & & & $61 \%-90 \%$ : Competente \\
\hline & & & $30 \%-60 \%$ : Aceptable \\
\hline
\end{tabular}




\begin{tabular}{|c|c|c|c|c|}
\hline $\begin{array}{l}\text { Parte II: } \\
\text {-Escuchar }(5 \%) \\
\text {-Entender }(5 \%) \\
\text {-Procesar }(5 \%) \\
\text {-Inferir }(5 \%)\end{array}$ & 20 pts. & $\begin{array}{l}\text { Parte II: } \\
\text {-Escuchar (5\%) } \\
\text {-Entender (5\%) } \\
\text {-Procesar (5\%) } \\
\text {-Inferir }(5 \%)\end{array}$ & & Menor a 30\%: No aceptable \\
\hline & $\begin{array}{l}\text { Total: } \\
100 \text { pts. }\end{array}$ & & Total: & \\
\hline & & & Porcentaje: & \\
\hline
\end{tabular}

Fuente: Buck (2001).

La cuarta etapa de este estudio consistió en el período de intervención. Éste involucró cinco tareas cooperativas (una por sesión) para la práctica de la comprensión auditiva, las cuales estuvieron subdivididas y realizadas como se muestra en la Tabla $\mathrm{N}^{\circ} 2$.

El último y quinto procedimiento aplicado consistió en la aplicación de un post test, para medir si hubo un mejoramiento en la comprensión auditiva. Éste fue de diferente temática, pero con la misma complejidad lingüística que el pre test. Constó de la misma estructura (dos secciones o partes), y al igual que en el pre test fueron evaluadas las características consideradas en el constructo de Buck (2001). El audio de la primera parte tuvo una duración de 01:02 minutos y era de la misma teleserie pero con diferentes personajes y contextos. Las oraciones de la segunda parte también fueron diferentes, pero siempre teniendo en cuenta la cultura brasileña y la misma complejidad lingüística.

Tabla $N^{\circ}$ 2. Distribución de tareas por período de intervención

\begin{tabular}{|l|l|l|}
\hline Período de intervención & \multicolumn{1}{|c|}{ Grupo Control } & \multicolumn{1}{c|}{ Grupo Experimental } \\
\hline $\begin{array}{l}\text { Tareas de pre audición } \\
\text { (15 minutos) }\end{array}$ & $\begin{array}{l}\text { Hubo tareas introductorias al } \\
\text { audio. De esta forma, los apren- } \\
\text { dientes se familiarizaron con el } \\
\text { contexto y el vocabulario que les } \\
\text { ayudó en la comprensión. }\end{array}$ & $\begin{array}{l}\text { Se hizo una introducción al video } \\
\text { foco de la tarea. Los aprendientes se } \\
\text { familiarizaron con el contexto y el } \\
\text { vocabulario que les ayudó en la com- } \\
\text { prensión (se llevó a cabo de manera } \\
\text { presencial). }\end{array}$ \\
\hline $\begin{array}{l}\text { Tareas durante la audición } \\
\text { (30 minutos) }\end{array}$ & $\begin{array}{l}\text { Los estudiantes debieron reu- } \\
\text { nirse en parejas y trabajar coo- } \\
\text { perativamente en la realización } \\
\text { de las diferentes actividades del } \\
\text { audio. }\end{array}$ & $\begin{array}{l}\text { Los estudiantes debieron entrar al } \\
\text { videoblog del curso y ver el video } \\
\text { presentado. Posteriormente, median- } \\
\text { videocomunicación, trabajaron } \\
\text { con un compañero en la realización } \\
\text { de actividades cooperativas sobre el } \\
\text { mismo video. Finalmente, escribie- } \\
\text { ron un texto en parejas y lo subieron } \\
\text { al foro para presentarlo al resto del } \\
\text { curso (se llevó a cabo de manera no } \\
\text { presencial). }\end{array}$ \\
\hline
\end{tabular}




\begin{tabular}{|c|c|c|}
\hline $\begin{array}{l}\text { Tareas de post audición } \\
\text { (10-15 minutos) }\end{array}$ & $\begin{array}{l}\text { Una vez que los aprendientes } \\
\text { llevaron a cabo las tareas ante- } \\
\text { riores, se pusieron en común } \\
\text { las dudas y comentarios. Se les } \\
\text { señalaron los aspectos en los } \\
\text { que debieron haber puesto más } \\
\text { atención para comprender el au- } \\
\text { dio y se respondieron todas sus } \\
\text { preguntas. }\end{array}$ & $\begin{array}{l}\text { Después de que los estudiantes } \\
\text { realizaron las tareas anteriores, hubo } \\
\text { una nueva sesión presencial. En ésta } \\
\text { se pusieron en común las dudas y } \\
\text { comentarios de los aprendientes en } \\
\text { relación a las tareas realizadas. Se les } \\
\text { señalaron los aspectos en los que de- } \\
\text { bieron haber puesto más atención y } \\
\text { se respondieron todas sus preguntas. }\end{array}$ \\
\hline
\end{tabular}

Fuente: elaboración propia.

\subsection{DISEÑO Y PROCEDIMIENTO}

Como ya se ha mencionado, tanto las tareas como el pre y post test tuvieron su fundamento teórico y de diseño en lo propuesto en el Constructo Estandarizado para la Comprensión Auditiva (Buck, 2001). La distribución de todos los instrumentos de recolección de datos y del período de intervención, tanto para el grupo control como para el grupo experimental, se muestran en la Tabla $\mathrm{N}^{\circ} 3$.

Tabla $N^{\circ}$ 3. Distribución de sesiones por período de intervención

\begin{tabular}{|l|l|l|}
\hline \multicolumn{1}{|c|}{ Sesiones } & Modalidad Grupo Control & Modalidad Grupo Experimental \\
\hline Cuestionario Inicial & Presencial & Presencial \\
\hline Pre Test & Presencial & Presencial \\
\hline Tarea 1: "Refrigerante Sukita” & Presencial & Presencial/ No Presencial \\
\hline Tarea 2: "Papo de Botequim” & Presencial & Presencial/ No Presencial \\
\hline Tarea 3: "Cerveja Polar" & Presencial & Presencial/ No Presencial \\
\hline Tarea 4: "Central do Brasil" & Presencial & Presencial/ No Presencial \\
\hline Tarea 5: "A Partilha" & Presencial & Presencial/ No Presencial \\
\hline Post Test / Cuestionario Final & Presencial & Presencial \\
\hline
\end{tabular}

Fuente: elaboración propia.

Las diferentes sesiones se efectuaron en el Centro de Estudos de Línguas (CEL) de la Universidad Estadual de Campinas, en una sala facilitada por esa casa de estudios para los propósitos de esta investigación. Ésta contaba con todos los implementos necesarios para reproducir los audios con buena calidad, mostrar las imágenes, dar explicaciones gramaticales, de léxico, fonéticas o de cualquier otro tipo y responder a las preguntas de los aprendientes. 


\section{RESULTADOS}

Después de la recolección de datos, se procedió al análisis de los resultados obtenidos en el pre y post test. Como primer paso, se realizó la prueba F de Fisher, para saber si los grupos eran homogéneos. Esta prueba permite calcular si las diferencias mostradas por las poblaciones en estudio son significativas y, por lo tanto, se pueden atribuir a cambios importantes en su comportamiento.

En la Tabla $\mathrm{N}^{\circ} 4$ aparecen los valores del pre test de ambos grupos.

Tabla $N^{\circ}$ 4. Prueba F de Fisher

\begin{tabular}{|c|c|c|}
\hline Aprendiente & Datos 1 & Datos 2 \\
\hline 1 & 30 & 35 \\
\hline 2 & 25 & 55 \\
\hline 3 & 20 & 40 \\
\hline 4 & 65 & 20 \\
\hline 5 & 20 & 70 \\
\hline 6 & 50 & 30 \\
\hline 7 & 20 & 35 \\
\hline 8 & 30 & 30 \\
\hline
\end{tabular}

Fuente: elaboración propia.

El valor obtenido corresponde a $\mathrm{p}=0,46$. A partir de éste, se concluye que ambos grupos no presentan diferencias significativas y se consideran homogéneos.

Luego de este procedimiento, se analizaron los resultados del pre y post test del grupo control y del experimental. Como ya fue señalado, ambas pruebas tenían dos secciones. Para su evaluación se consideraron 10 puntos por cada respuesta correcta, que en total sumaban 100 puntos (100\%), tal como se observa en la Tabla $\mathrm{N}^{\circ} 1$.

Como se trataba de pruebas de comprensión, se les señaló a los estudiantes que podían responder tanto en portugués como en español (su lengua materna). Esto no tendría ningún tipo de descuento en el puntaje total, así como tampoco lo tendrían los posibles errores gramaticales u ortográficos que ellos cometieran en la lengua meta.

De acuerdo a la pregunta de investigación e hipótesis planteada, las siguientes tablas muestran los resultados obtenidos por cada sujeto participante en este estudio. En primer lugar se presentan los datos del grupo control para el pre y el post test en la Tabla $\mathrm{N}^{\circ} 5$. 
Tabla $N^{\circ} 5$. Resultados pre y post test del Grupo Control

\begin{tabular}{|c|c|c|}
\hline Aprendiente & Pre test & Post test \\
\hline 1 & 30 & 80 \\
\hline 2 & 25 & 15 \\
\hline 3 & 20 & 30 \\
\hline 4 & 65 & 80 \\
\hline 5 & 20 & 95 \\
\hline 6 & 50 & 60 \\
\hline 7 & 20 & 65 \\
\hline 8 & 30 & 65 \\
\hline Promedio & 32,5 & 61,2 \\
\hline
\end{tabular}

Fuente: elaboración propia.

Se observa que la mayoría de los aprendientes mejoraron en la comprensión auditiva de portugués como LE. Sólo un participante (2) disminuyó su desempeño inicial en 10 puntos. Los demás mejoraron entre los 10 y los 75 puntos de diferencia.

Debido al tamaño de la muestra, se utilizó la prueba estadística de t-student para muestras emparejadas, la cual consiste en medir si las medias de dos grupos son estadísticamente significativas una de otra. Los resultados obtenidos al comparar el desempeño de los aprendientes en el pre y post test arrojan un valor $\mathrm{p}=0,02$, considerado estadísticamente significativo, ya que $\mathrm{p}<0,05$.

En la Tabla $N^{\circ} 6$ se observan los resultados que obtuvo el grupo experimental.

Tabla $N^{\circ}$ 6. Resultados pre y post test del Grupo Experimental

\begin{tabular}{|c|c|c|}
\hline Aprendiente & Pre test & Post test \\
\hline 1 & 35 & 50 \\
\hline 2 & 55 & 95 \\
\hline 3 & 40 & 65 \\
\hline 4 & 20 & 80 \\
\hline 5 & 70 & 65 \\
\hline 6 & 30 & 75 \\
\hline 7 & 35 & 80 \\
\hline 8 & 30 & 90 \\
\hline Promedio & 39,3 & 75 \\
\hline
\end{tabular}

Fuente: elaboración propia. 
Al igual que en el grupo control, en el grupo experimental sólo un participante mostró una baja en su puntaje (5), disminuyendo 5 puntos en relación al puntaje inicial obtenido. En consecuencia, la mayoría de los alumnos mejoró su desempeño en la comprensión auditiva de un texto oral. No obstante, el grupo experimental mostró un mayor aumento en el aprendizaje. La prueba t-student de muestras emparejadas también fue aplicada para este grupo. Se obtuvo, así, un valor $\mathrm{p}=0,002$. Este resultado también es considerado estadísticamente significativo $(\mathrm{p}<0,05)$. Un análisis más detallado queda de manifiesto a continuación.

Si se analizan ambos grupos de manera conjunta, se observa que la distancia entre ellos creció después del período de intervención: pasó de ser de 6,8 puntos en el pre test a 13,8 puntos en el post test, es decir, 7 puntos de diferencia, tal como se observa en la Tabla $\mathrm{N}^{\circ} 7$.

Tabla $N^{\circ}$ 7. Diferencia de puntajes Grupo Control y Grupo Experimental

\begin{tabular}{|c|c|c|c|}
\hline & Pre test & Post test & \\
\hline Prom. Grupo Control & 32,5 & 61,2 & Dif. 28,7 \\
\hline Prom. Grupo Experimental & 39,3 & 75 & Dif. 35,7 \\
\hline & Dif. 6,8 & Dif. 13,8 & \\
\hline
\end{tabular}

Fuente: elaboración propia.

Asimismo, los puntajes dentro de cada grupo advierten un incremento de 28,7 puntos para el grupo control, mientras que el grupo experimental aumentó en 35,7 puntos de su desempeño inicial, lo cual se muestra más claramente en el siguiente gráfico.

Gráfico $N^{\circ} 1$. Resultados generales pre y post test

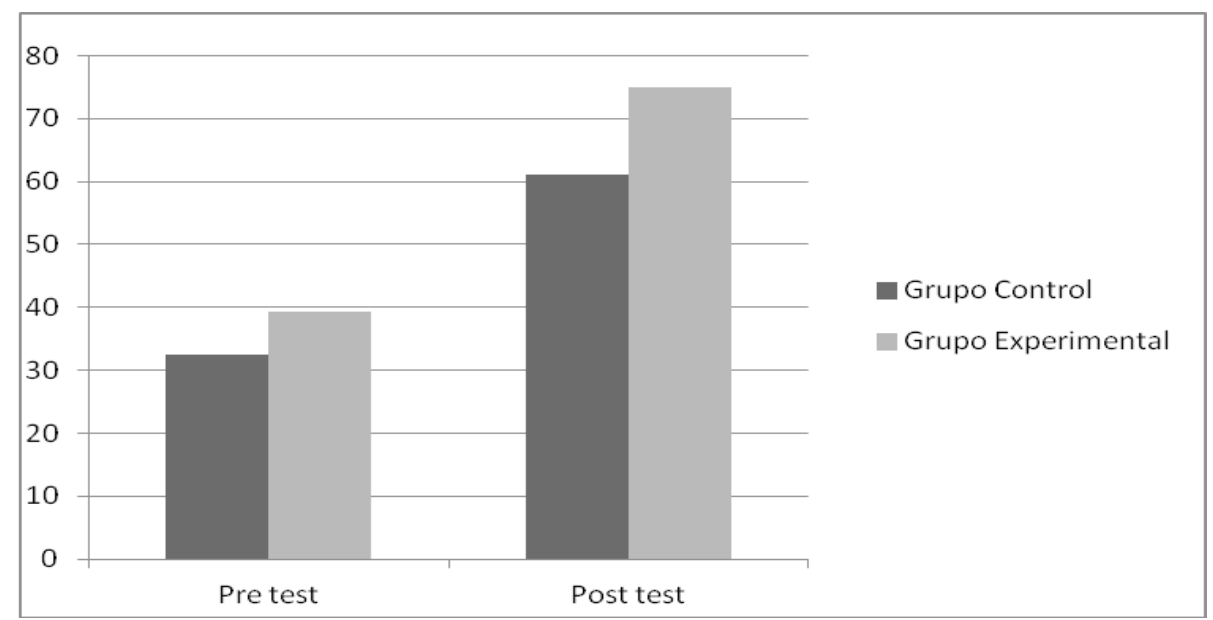

Fuente: elaboración propia. 
Con los resultados obtenidos en ambos grupos, se puede, de esta forma, negar la hipótesis nula del presente estudio. Sin embargo, y debido al reducido tamaño de la muestra, los resultados no son generalizables y sólo señalan una tendencia al mejoramiento de la comprensión auditiva de hispanohablantes aprendientes de portugués como LE, en relación a lo planteado en el Constructo Estandarizado para la Comprensión Auditiva (Buck, 2001).

\section{CONCLUSIONES}

El avance alcanzado por la operacionalización del modelo de enseñanza aquí propuesto evidencia que las nuevas tecnologías se constituyen en un recurso favorable para promover el desarrollo de la comprensión auditiva, ya que se pueden presentar estímulos orales y visuales de manera conjunta, auténticos y contextualizados, como sería en una interacción real cara a cara (Meskill, 1996). Es por este motivo que la problemática abordada en el presente trabajo se constituye en una contribución en el área del aprendizaje de la comprensión auditiva, puesto que evidenció empíricamente la efectividad de esta metodología para la enseñanza de la habilidad.

Los estudios revisados hasta ahora han trabajado con aplicaciones tecnológicas para promover el desarrollo de esta destreza, pero no las han confrontado con el modo tradicional de enseñanza (escuchar un audio durante una clase presencial), como en el presente trabajo. Por otra parte, estas investigaciones analizan, en su mayoría, otras lenguas y con otro tipo de muestra (aprendientes de portugués nativos de francés o inglés, como en los estudios conducidos por Santos (2008) y De Souza (2003), respectivamente), por lo que se hace necesario continuar estudiando en esta línea y considerar las especificidades de lenguas con tantas similitudes, como es el caso de hispanohablantes aprendientes de portugués como LE.

De acuerdo con los resultados obtenidos, el uso de los recursos tecnológicos como el videoblog, el foro y la videocomunicación parecen ser herramientas beneficiosas para el mejoramiento de la comprensión auditiva, con un incremento de 35,7 puntos del desempeño inicial del grupo experimental. Siete puntos sobre el valor final del grupo control (28,7 puntos). Esto puede deberse, en parte, a que los aprendientes tuvieron acceso a soporte audiovisual, el cual puede haber facilitado la comprensión de los textos orales de las diferentes tareas. No obstante, cabe señalar que el reducido tamaño de la muestra y la corta duración del período de intervención (cinco sesiones), no permiten formular conclusiones definitivas relacionadas con el mejoramiento de la comprensión auditiva mediante el uso de tecnología versus la forma tradicional. Por tal razón, es imprescindible seguir trabajando e investigando al respecto, con estudios más prolongados en el tiempo y muestras de mayor tamaño. Pese a lo anterior, se puede afirmar que los resultados sí muestran una tendencia positiva hacia el uso de tecnología para la práctica de esta destreza, como también se demuestra en los estudios de Santos (2008), De Souza (2003), Mueller (1980) y Omaggio (1980) (Cit. en Meskil, 1996), todos señalados anteriormente.

Asimismo, se debe indicar que la enseñanza de una nueva lengua con materiales auténticos, ya sea audio o video, y el uso de tareas cooperativas, trae consigo un mejoramiento de la comprensión auditiva, en el sentido que propone el Constructo Estandarizado para la Comprensión Auditiva (Buck, 2001). Es decir, comprender un texto oral es procesar material oral auténtico, en un contexto y en tiempo real, entender la información linguiística que en 
él aparece, y a partir de ahí, formular las posibles inferencias implicadas en el contenido del texto. Esto, aplicado a aprendientes de nivel básico (especialmente hispanohablantes, que según Pinto (2010), Azevedo (1978) y Holton (1954) no son considerados propiamente principiantes), favorece que desde un principio vayan internalizando diferentes aspectos de la lengua meta (como segmentación de palabras, reconocimiento de palabras nuevas, tonos, marcadores pragmáticos, implícitos, entre otros), y que puedan interactuar de manera más proficiente en la nueva lengua y cultura. Pese a todo lo anterior y a los resultados encontrados, estadísticamente significativos, hay que tener en cuenta que los estudiantes se encontraban en inmersión, por lo cual estaban expuestos a un contexto donde el portugués era la lengua de comunicación. Debido a esto, es necesario investigar cuales serían los resultados en un contexto donde el portugués no sea la lengua oficial, como puede ser el caso de Chile.

De esta forma, se espera que lo planteado aquí pueda servir de base para futuras investigaciones, tanto en el área de CMC como de portugués como LE. Además, se espera que sea un aporte para el mayor y mejor entendimiento en la enseñanza de la comprensión auditiva de hispanohablantes aprendientes de portugués como lengua extranjera.

\section{REFERENCIAS BIBLIOGRÁFICAS}

Adrián, M. y Gros, B. (2004). Estudio sobre el uso de los foros virtuales para favorecer las actividades colaborativas en la enseñanza superior. Teoría de la Educación: Educación y Cultura en la Sociedad de la Información, (5), 11-21. Recuperado el 6 de mayo de 2012 desde http://dialnet. unirioja.es/servlet/articulo?codigo $=1048868$

Albuquerque, A. y Esperança, J. (2010). El valor económico del portugués: Lengua de conocimiento con influencia global. Real Instituto Elcano, (26), 1-11. Recuperado el 10 de abril de 2012 desde http://www.realinstitutoelcano.org/wps/portal/rielcano/contenido?WCM_GLOBAL_ CONTEXT=/elcano/elcano_es/zonas_es/dt26-2010

Almeida Filho, J. (2004). Questões da interlíngua de aprendizes de português a partir e com a interposição do espanhol (língua muito próxima). Em A. Simões, A. M. Carvalho e L. Wiedemann (Orgs.), Português para falantes de espanhol (pp. 183-191). Campinas: Pontes Editores.

Anderson, A. \& Lynch, T. (1988). Listening. New York: Oxford University Press.

Andrighetti, G. (2009). A elaboração de tarefas de compreensão oral para o ensino de português como língua adicional em níveis iniciais. Tese de Mestrado em Lingǘstica Aplicada. Universidade Federal de Rio Grande do Sul, Brasil.

Arango, M. (2003). Foros virtuales como estrategia de aprendizaje. Revista Debates Latinoamericanos, (2). Recuperado el 6 de mayo de 2012 desde http://www.rlcu.org.ar/revista/ numeros/02-02-Abril-2004/documentos/Arango.pdf

Azevedo, M. (1978). Identifying Spanish Interference in the Speech of Learners of Portuguese. The Modern Language Journal, vol.6 (1), 18-23.

Bison, A. (1994). Características da interação em contexto de ensino regular e em contexto de ensino interdisciplinar de português língua estrangeira: Um estudo comparativo. Tese de Mestrado em Lingüística Aplicada. Universidade Estadual de Campinas, Brasil.

Brown, H. D. (2001). Teaching by Principles: An Interactive Approach to Language Pedagogy. New York: Pearson Education.

Brown, S. (2006). Listening. New York: Cambridge University Press.

Buck, G. (2001). Assessing Listening. Cambridge: Cambridge University Press.

Córdova, P., Coto, R. y Ramírez, M. (2005). La comprensión auditiva: Definición, importancia, 
características, procesos, materiales y actividades. Actualidades Investigativas en Educación, vol.5 (1), 1-17. Recuperado el 22 de diciembre de 2011 desde http://redalyc.uaemex.mx/pdf/447/44750107.pdf

De Souza, R. (2003). Telecolaboração e divergência em uma experiência de aprendizagem de português e inglês como línguas estrangeiras. Revista Brasileira de Linguística Aplicada, vol.3 (2), 73-96.

Doughty, C. \& Long, M. (2003). Optimal Psycholinguistic Environments for Distance Foreign Language Learning. Language Learning and Technology, vol.7 (3), 50-80. Retrieved on September 9, 2011 from http://ltt.msu.edu/vol7num3/doughty/default.html

Estaire, S. (2009). El aprendizaje de lenguas mediante tareas: De la programación al aula. Madrid: Editora Edinumen.

Ferreira, A. (2007). Bases teóricas para un modelo b-learning de enseñanza de lenguas extranjeras. En J. Sánchez (Ed.), Nuevas ideas en informática educativa (pp. 272-296). Santiago de Chile: LOM Ediciones.

García, J., Ferreira, A. y Morales, S. (2012). Autonomía en el aprendizaje de lenguas extranjeras en contextos de enseñanza mediados por la tecnología. Revista Onomázein, vol.25 (1), 15-50.

Holton, J. (1954). Portuguese for Spanish Speakers. Hispania, vol.37 (4), 446-452.

Jauregi, K., Canto, S. y Gómez, J. (2010). Interacción virtual a través de la videocomunicación y mundos virtuales: Dos estudios piloto. MarcoELE. Revista de Didáctica ELE, (11), 1-17.

Johnson, D. y Johnson, R. (1999). Aprender juntos y solos. São Paulo: Carrera Docente.

Johnson, K. (2008). Aprender y enseñar lenguas extranjeras: Una introducción. México D.F.: Fondo de Cultura Económica.

Jones, L. C. (2008). Listening Comprehension Technology: Building the Bridge from Analog to Digital. Calico Journal, vol.25 (3), 400-419.

Lamy, M. N. \& Hampel, R. (2007). Online Communication in Language Learning and Teaching. Hampshire: Palgrave Macmillan.

Livingstone, K. y Ferreira, A. (2010). La efectividad de un modelo metodológico mixto para la enseñanza-aprendizaje de español como lengua extranjera. Boletín de Filología, vol.44 (2), 89-118.

Lynch, T. (2002). Listening: Questions of Level. In R. Kaplan (Ed.), The Oxford Handbook of Applied Linguistics (pp. 39-48). New York: Oxford University Press.

Meskill, C. (1996). Listening Skills Development through Multimedia. Journal of Educational Multimedia and Hypermedia, vol.5 (2), 179-201.

Morales, S. y Ferreira, A. (2008). La efectividad de un modelo de aprendizaje combinado para la enseñanza del inglés como lengua extranjera: Estudio empírico. RLA. Revista de Lingüística Teórica y Aplicada, vol.46 (2), 95-118.

Ornelas, D. (2007). El uso del foro de discusión virtual en la enseñanza. Revista Iberoamericana de Educación, vol.4 (44), 1-5. Recuperado el 8 de mayo de 2012 desde http://www.rieoei.org/1900.htm

Peris, E. (1991). La didáctica de la comprensión auditiva. Revista Cable, (8), 1-11. Recuperado el 11 de octubre de 2011 desde http://www.upf.edu/pdi/dtf/ernesto.martin/archivos/articulos/ didactica_comprension_auditiva.pdf

Pica, T., Kanagy, R. \& Falodun, J. (1993). Choosing and Using Communicative Tasks for Second Language Instruction. In G. Crookes \& S. Gass (Eds.), Tasks in a Pedagogical Context (pp. 9-34). Clevedon: Multilingual Matters.

Pinto, P. F. (2010). Oralidade na aula de português para hispanofalantes. Límite, (4), 141-151.

Quintanilla, A. y Ferreira, A. (2010). Habilidades comunicativas en L2 mediatizadas por la tecnología en el contexto de los Enfoques por Tareas y Cooperativo. Estudios Pedagógicos, vol.36 (2), 213-231.

Santos, L. (2008). Ensino e aprendizagem de português para estrangeiros in-tandem em contexto virtual: Primeiros passos de um projeto franco-brasileiro. I SIMELP - Simpósio Mundial de Estudos de Língua Portuguesa. São Paulo, Brasil.

Sato, E. (2011). A compreensão oral nos cadernos de língua estrangeira modernas - inglês 
no estado de São Paulo. Tese de Mestrado em Lingüística Aplicada. Universidade Estadual de Campinas, Brasil.

Trujillo Sáez, F. (2002). Aprendizaje cooperativo para la enseñanza de la lengua. Revista Publicaciones de la Facultad de Educación y Humanidades Campus de Melilla, (32), 147-162. Recuperado el 10 de mayo de 2012 desde http://meteco.ugr.es/lecturas/cooperacion.pdf

Vandergrift, L. (2011). Second Language Listening: Presage, Process, Product and Pedagogy. In E. Hinkel (Ed.), Handbook of Research in Second Language Teaching and Learning. Vol. 2 (pp. 455-471). New York: Routledge. (2007). Recent Developments in Second and Foreign Language Listening Learning Comprehension Research. Cambridge Journals, vol.40 (3), 191-210. (24), 3-25. . (2004). Listening to Learn or Learning to Listen? Annual Review of Applied Linguistics,

Vine, A. y Ferreira, A. (2012). Mejoramiento de la competencia comunicativa en español como lengua extranjera a través de la videocomunicación. RLA. Revista de Lingüística Teórica y Aplicada, vol.50 (1), 139-160.

White, C. (2003). Language Learning in Distance Education. Cambridge: Cambridge University Press.

Widdsowson, H. G. (1991). O ensino de línguas para a comunicação. Campinas: Pontes Editores. 